[0212-7199 (2006) 23: 12; pp 593-595] ANALES DE MEDICINA INTERNA Copyright (C) 2006 ARAN EDICIONES, S.L.

AN. MED. INTERNA (Madrid) Vol. 23, N. ${ }^{\circ} 12$, pp. 593-595, 2006

\title{
Pileflebitis secundaria a diverticulitis
}

\author{
S. CASALLO BLANCO, A. I. MUÑOZ RUIZ, F. MARCOS SÁNCHEZ, \\ L. DE MATÍAS SALCES, J. BLANCO GONZÁLEZ', C. CASTAÑEDA BERGAMÍN' \\ Servicios de Medicina Interna y de ${ }^{1}$ Digestivo. Hospital Nuestra Señora del Prado. \\ Talavera de la Reina. Toledo
}

\begin{abstract}
RESUMEN
Presentamos el caso de un varón de 52 años, con antecedentes de cólicos renoureterales que había consultado por un episodio de similares características, acompañado de fiebre y elevaciones de la GGT y de la fosfatasa alcalina. Una ecografía abdominal mostró hepatoesplenomegalia y un material ecogénico en el eje esplenoportal, sugerente de trombosis. La TAC abdominal demostró trombosis de la vena mesentérica inferior, continuándose por la vena esplénica hasta el origen proximal de la vena porta. También se observaron numerosos divertículos. Se sospechó que el paciente presentaba una diverticulitis aguda complicada con trombosis vascular, por lo que se decidió la realización de cirugía, que confirmó la existencia de diverticulitis sin perforación ni abscesos, flebitis con pileflebitis de la arteria mesentérica inferior hasta su unión con la vena esplénica, con salida de pus al seccionar la vena. Se realizó ligadura de la vena mesentérica inferior e intervención de Hartman con ligadura del munón rectal y colostomía. Además se indicó tratamiento antibiótico de amplio espectro y anticogulación, siendo la evolución favorable.

Posteriormente comentamos algunos aspectos etiológicos, clínicos, diagnósticos y terapéuticos de esta infrecuente complicación de la diverticulitis.
\end{abstract}

PALABRAS CLAVES: Pileflebitis. Diverticulitis. Trombosis de la porta.

Casallo Blanco S, Muñoz Ruiz AI, Marcos Sánchez F, de Matías Salces L, Blanco GonzálezJ, Castañeda Bergamín C. Pileflebitis secundaria a diverticulitis. An Med Interna (Madrid) 2006; 23: 593-595.

\begin{abstract}
A case of a 52 year-old-male, with past medical history of renoureteral crisis and recurrent episodes of abdominal pain, is presented. The patient presented to the Emergency Department with abdominal pain (similar to previous episodes), fever and abnormal liver function test (marked elevation of gammaglutamyltranspeptidase and alkaline phosphatase). An abdominal ultrasound was performed showing hepatomegaly, and enlarged spleen and an echogenic material that suggested a thrombosis. A CT scan confirmed the thrombosis of the inferior mesenteric vein extending up to the splenic vein and the portal vein. It also showed a large number of diverticulum. Surgery was performed in order to rule out an acute diverticulitis. A phylephlebitis, infective suppurative thrombosis of the portal vein and its branches (inferior mesenteric vein and splenic vein) was found due to an acute diverticulitis with neither perforation nor abscess. A ligature of the inferior mesenteric vein and a Hartmann procedure with resection of the diseased colon, and end colostomy and creation of a rectal stump, were performed. A favourable outcome was obtained with antibiotics and anticoagulation.

Some aspects of the aetiology, symptoms, diagnosis and treatment of this unusual complication of diverticulitis are also presented.
\end{abstract}

KEY WORDS: Pylephlebitis. Diverticulitis. Thrombosis of the portal vein.

\section{INTRODUCCIÓN}

La pileflebitis o tromboflebitis séptica de la vena porta es un proceso infeccioso cuya incidencia ha ido en aumento en los últimos años, debido especialmente, a un incremento en su detección por medio de las técnicas de imagen, tales como la ultrasonografía y la tomografía axial computerizada (TAC) (1).

La tromboflebitis séptica de la vena porta puede complicar la sepsis intrabdominal de cualquier etiología, aumen- tando la morbilidad y mortalidad de manera significativa.

A pesar del desarrollo de nuevos antibióticos y anticoagulantes, la tasa de mortalidad en las diferentes series se mantiene alrededor del 35\% (1). La medida más importante para conseguir la reducción de estas cifras de mortalidad sería poder establecer un diagnóstico precoz de la enfermedad, localizar el foco infeccioso causante de la trombosis portal e iniciar el tratamiento adecuado.

Presentamos el caso de un paciente con pileflebitis secundaria a diverticulitis.

Trabajo aceptado: 27 de junio de 2006

Correspondencia: Fernando Marcos Sánchez. C/ Gregorio, 1. 45600 Talavera de la Reina (Toledo). 


\section{CASO APORTADO}

Varón de 52 años con antecedentes personales de cólicos nefríticos de repetición, acude a urgencias por dolor en fosa renal izquierda siendo diagnosticado de posible crisis renoureteral, indicando tratamiento con ketorolaco y metamizol.

Diez días más tarde acude nuevamente a urgencias por fiebre, dolor en fosa renal izquierda e hipogastrio, disuria y polaquiuria.A la exploración física presentaba mal estado general, temperatura de $38^{\circ}$ y en el abdomen destacaba dolor a la palpación en fosa iliaca izquierda e hipogastrio con defensa, el resto de la exploración física fue normal.

En cuanto a las pruebas complementarias, destacaba en el hemograma la existencia de 17.200 leucocitos, con desviación a la izquierda, plaquetas de 415.000, con serie roja normal y con un estudio de coagulación con una actividad de protrombina de 70,7\%.

En la bioquímica destacaba elevación de la gammaglutamiltranspeptidasa (GGT): $334 \mathrm{U} / 1$ y fosfatasa alcalina (FA) $255 \mathrm{UI} / \mathrm{l}$; bilirrubina, creatinina, glucosa, proteínas totales, iones, amilasa, GPT, gammaoxalatotransaminasa (GOT) fueron normales. La radiografía de tórax fue normal y el electrocardiograma presentaba una taquicardia sinusal, sin alteraciones de la repolarización. En el sistemático de orina se objetivaban indicios de eritrocitos, bilirrubina (+), cuerpos cetónicos (+) y en el sedimento 2 hematíes campo.

Se realizó una ecografía abdominal, objetivando hígado aumentado de tamaño (18 centímetros), de ecogenicidad homogénea, sin lesiones ocupantes de espacio, esplenomegalia homogénea de 15,4 $\mathrm{cm}$ y material ecogénico en eje esplenoportal (en la unión de venas esplénica y mesentérica superior) (Fig. 1), que no se descartaba que correspondiera con un pequeño trombo no oclusivo en el origen de la vena porta. Ante este hallazgo se realizó una TAC abdominal donde se objetivaba trombosis de la vena mesentérica inferior (VMI) en todo su trayecto, que se continuaba por vena esplénica hasta el origen proximal de la vena porta. Distalmente se observaba engrosamiento de pequeñas venas sigmoideas tributarias de VMI y numerosos diverticulos en sigma (probablemente engrosado), sin que se pudiera valorar con fiabilidad signos francos de diverticulitis aguda debido a las alteraciones a nivel de la grasa regional (Fig. 2).

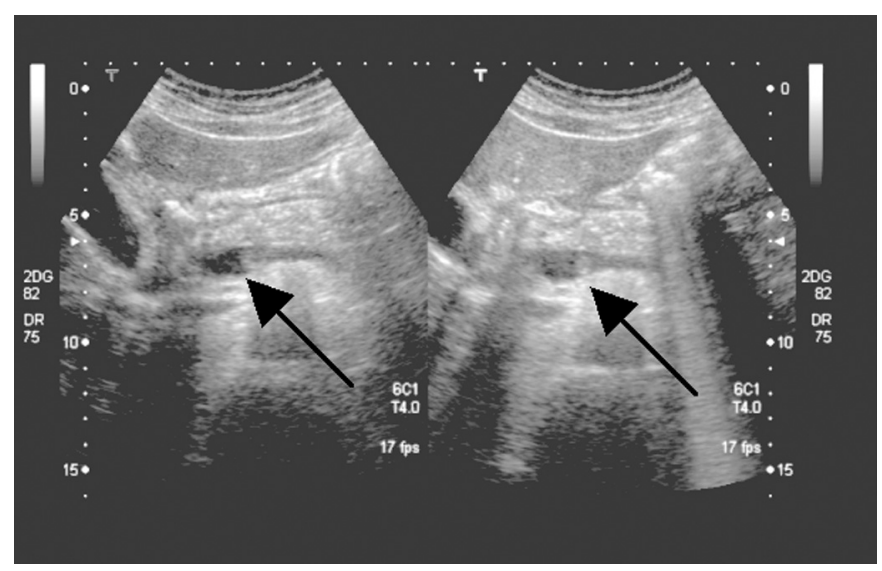

Fig. 1. Material ecogénico en el eje esplenoportal (en la unión de las venas esplénica y mesentérica superior).

Estos hallazgos sugerían que probablemente nuestro paciente presentaba una diverticulitis aguda complicada con trombosis de la vena mesentérica inferior y de la porta, por lo que se avisó a cirugía decidiendo intervención quirúrgica urgente. En la laparotomía se observaron signos de diverticulitis aguda sin perforación ni absceso, flebitis con pileflebitis de la arteria mesentérica inferior hasta su unión con la vena esplénica, salida de pus a nivel de la sección de la vena; realizándose ligadura de la vena mesenterica inferior proximal a su unión con la vena esplénica más intervención de Hartman con

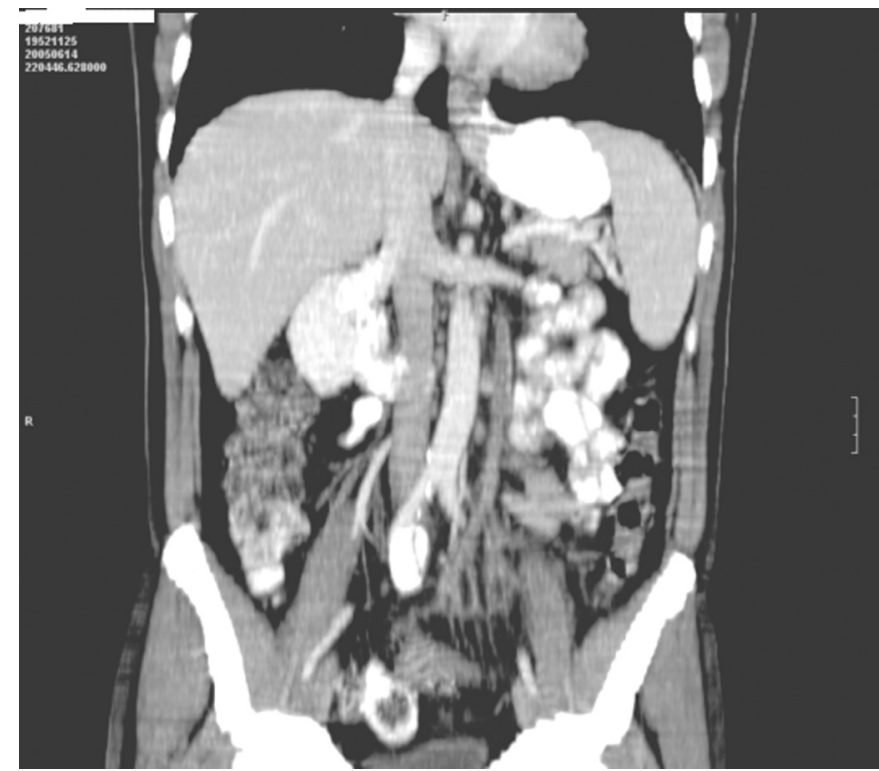

Fig. 1. TAC abdominal (reconstrucción). Trombosis de la vena mesentérica inferior en todo su trayecto, que se continúa por la vena esplénica hasta el origen proximal de la vena porta.

ligadura del muñón rectal y colostomía terminal en fosa iliaca izquierda. El postoperatorio transcurrió sin incidencias, iniciándose tratamiento con anticoagulación oral y antibiótioterapia de amplio espectro con ampicilina, gentamicina y metronidazol, presentando buena evolución, siendo dado de alta a las tres semanas.

\section{DISCUSIÓN}

La pileflebitis puede complicar cualquier infección abdominal o pélvica que ocurra en la región drenada por el sistema venoso portal, especialmente diverticulitis, como en nuestro caso, y menos frecuentemente apendicitis, colangitis o enfermedad inflamatoria intestinal (2). Aunque la causa más común de pileflebitis es la diverticulitis, la pileflebitis es una complicación rara de la diverticulitis. Algunos casos se asocian a alteraciones de la coagulación como los estados de hipercoagulabilidad, déficit de factores, enfermedades malignas como el carcinoma hepatocelular o síndrome de inmunodeficiencia adquirida $(3,4)$.

La presencia de un foco infeccioso abdominal en un territorio que drene a la vena porta provoca la salida al torrente vascular de una serie de células inflamatorias como linfocitos, leucocitos polimorfonucleares y macrófagos que producen un ambiente procoagulante muy propicio para desarrollar la trombosis portal. En ocasiones estos émbolos sépticos son enviados al hígado, dando lugar a la formación de abscesos hepáticos. La mayor parte de los abscesos se presentan en el lóbulo hepático derecho debido al drenaje preferencial laminar secundario de las venas mesentéricas superiores. La probabilidad de desarrollo de abscesos hepáticos en el contexto de una pileflebitis puede estar entre el 8 y el $10 \%$ de los casos (5).

La pileflebitis puede dar lugar a complicaciones importantes como trombosis de la vena porta y de sus ramas. En algunos estudios hasta en un 34\% de los casos estaba afectada la vena mesentérica superior, lo cual pueda dar lugar a una 
isquemia intestinal. La hipertensión portal puede ser otra complicación de esta enfermedad $(1,6)$. El microorganismo más comúnmente aislado en esta enfermedad es el Bacteroides fragilis, seguido por bacilos aerobios Gram negativos como el E. coli y los Estreptococos aerobios. Según las series, en un $23-88 \%$ de casos existe bacteriemia, generalmente polimicrobiana (7). Los estreptococos, estafilococos y el citrobacter también se han descrito, pero los enterococos son una causa infrecuente de esta enfermedad.

La pileflebitis puede tener un inicio y una evolución clínica diversa: puede presentarse en un paciente casi asintomático hasta encontrarse formas graves que pueden llegar al shock séptico con insuficiencia hepática. La fiebre y el dolor abdominal son los signos más frecuentes. Según las series su frecuencia varia entre el $75-100 \%$ de los casos $(1,2)$. Debe sospecharse en presencia de ictericia, alteraciones de la coagulación y aumento de las enzimas hepáticas como la fosfata alcalina y las transaminasas (8).

El rango de edad de los pacientes afectados es ancha (6 a 75 años), que pueden ser influenciados parcialmente por el número de pacientes con apendicitis como etiología inicial. La edad media de los pacientes con pileflebitis es entorno a los 45 años (1). El diagnóstico de la pileflebitis requiere la demostración de trombosis venosa portal o existencia de gas en el sistema portal generalmente acompañada de bacteriemia en un paciente febril. Dado que es una entidad poco frecuente, con signos y síntomas inespecíficos, el diagnóstico con frecuencia se retrasa empeorando así el pronóstico de la enfermedad, siendo el diagnóstico precoz de vital importancia. Antes del advenimiento de los medios diagnósticos por imágenes, éste se realizaba de forma incidental intraoperatoria o en estudios de autopsias. Las técnicas más empleadas actualmente son la ecografía, la TAC y el eco-doppler. La imagen más frecuente es la presencia de material ecogénico en el interior de la luz portal y de la vena mesentérica, lo cual puede ser utilizado para el control evolutivo de la enfermedad, especialmente para detectar la recanalización del territorio portal y el desarrollo de cavernomatosis y/o de hipertensión portal. La presencia de aire intraluminal suele corresponder a un estadio avanzado de la pileflebitis y es signo de mal pronóstico. La TAC puede mostrar la trombosis o, menos frecuentemente gas portal y ayudar a definir el foco infeccioso abdominal así como para detectar el desarrollo de abscesos hepáticos (9). La resonancia magnética nuclear no se ha empleado extensamente en el diagnóstico de la pileflebitis ya que con la ecografía y la TAC obtenemos el diagnóstico.

El manejo de la pileflebitis se basa en la antibioterapia de amplio espectro, en el tratamiento quirúrgico en los casos de focos infecciosos intrabdominales y en la terapia anticoagulante y trombolítica, además del soporte hemodinámico. El tratamiento empírico con antibióticos de amplio espectro debe instaurarse tan pronto como sea posible, teniendo en cuenta el tipo de microflora mixta, mientras se obtiene el resultado de los cultivos, por lo que se debe combinar varios antibióticos recomendando: ampicilina $2 \mathrm{~g} / 4$ horas, gentamicina 1.5 $\mathrm{mg} / \mathrm{kg}$ y metronidazol $500 \mathrm{mg} / 8$ horas. La duración del tratamiento varía entre 2 y 4 semanas. En caso de que existan abscesos hepáticos debe prolongarse hasta 6 semanas (1).

El papel de la anticoagulación es controvertido, ya que no existen estudios concluyentes, parece haber efectos beneficiosos en los casos de trombosis portal extensa o en situaciones de clara progresión documentada por estudios radiológicos, en pileflebitis supurativa o después de una resección intestinal por isquemia secundaria a la trombosis portal (10). La terapia trombolítica es otra alternativa recomendada por diferentes autores.

Si se presentan abscesos hepáticos como complicación de la pileflebitis, el drenaje percutáneo junto con la antibioterapia es la modalidad terapéutica que exhibe los mejores resultados (supervivencia global de 90\%). Otro planteamiento es el drenaje abierto de la rama portal comprometida por una pileflebitis supurativa, colocando un drenaje intraluminal para facilitar la salida del contenido purulento al exterior. Esta situación es observada, con buenos resultados en el caso de trombosis portal idiopática extensa, en lugar del tratamiento antibiótico y trombolítico. Puede utilizarse también el abordaje percutaneo de la vena porta en casos de pileflebitis supurativa. A pesar de todo la mortalidad es elevada (11-32\%) (7).

\section{Bibliografía}

1. Plemmons RM, Dooley DP, Longfield RN. Septic thrombophebitis of the portal vein (pylephebitis): Diagnosis and management in the modern era. Clin Infect Dis 1995; 21: 1114-20.

2. Singh P, Yadav N, Visvalingam V, Indaram A, Bank S. Pylephebitis Diagnosis and management. Am J Gastroenterol 2001; 96: 1312-3.

3. Etienne M, Guiet I, Abboud P, Pons JL, Jacquot S, Caron F. Fusobacterium nucleatum hepatic abscess with pylephlebitis associated with idiopathic CD4+ T lymphocytopenia. Clin Infect Dis 2001; 32: 326-8.

4. Sillero JM, Calvet X, Musulen E, Díaz-Ruiz MJ, Tolosa C, Pardo A. Idiopathic pylephebitis and idiopathic sclerosing peritonitis in a man with protein S deficiency. J Clin Gastroenterol 2001; 32: 262-5

5. McDonald AP, Howard RJ. Pyogenic liver abscess. World J Surg 1980; 4: 369-80.
6. Chang TN, Tang L, Keller K, Harrison MR, Farmer DL, Albanese CT. Pylephebitis, portal-mesenteric thrombosis, and multiple liver abscesses owing to perforated appendicitis. J Pediatr Surg 2001; 36: E19

7. Baril N, Wren S, Radin R, Ralls P, Stain S. The role of anticoagulation in pylephebitis. Am J Surg 1996; 172: 449-53

8. Tung JY, Johnson JL, Liacouras CA. Portal-mesenteric pylephebitis with hepatic abscess in a patient with Crohn's disease treated successfully with anticoagulation and anthibiotics. J Pediatr Gastroenterol Nutr 1996; 23: 474-82.

9. Balthazar EJ, Gollapudi P. Septic thrombophebitis of the mesenteric and portal veins: CT imaging. J Comput Assist Tomogr 2000; 24: 755-60.

10. Rodríguez O, Plaza V, Castro O, Oltra R. Pileflebitis y anticoagulación. Med Intensiva 2002; 26: 136-136. 